\title{
Serum level of Alarin in Patients with Androgenetic Alopecia
}

A.M.Hamed, G.M.Abdel Khalik and M.G.Abdel Fatah

Dermatology, Venereology and Andrology, Dept., Faculty of Medicine, Benha Univ., Benha, Egypt

E-Mail:Marwagamal889@gmail.com

\begin{abstract}
Background: With androgenetic alopecia, hair follicle size decreases dramatically, which may be linked to the death of hair follicle stem cells or progenitor cells in the scalp. To far, scientists believe that AGA is caused by two things: a hereditary predisposition and androgens. In the last several decades, obesity and the metabolic syndrome have spread like wildfire across the globe, posing serious health and economic issues. According to some reports, Alarin is linked to increased caloric intake and weight gain. However, it's unclear if high levels of Alarin in the blood correlate with insulin resistance or the metabolic syndrome. Recent research shows a connection between AGA and cardiovascular disease (CVD), diabetes mellitus and hypertension dating back to the early 1990s (HT). The study's goal was to find out whether there was a link between high levels of serum alarin and androgenic alopecia, as well as obesity and the metabolic syndrome. Methods: The research was carried out at the outpatient clinic of the Benha University Hospitals' Dermatology and Andrology Department in the year 2020. There were 80 people in all, divided into two groups: First, there were 50 patients with androgenic alopecia (Hamilton-Norwood grades I-VII) and 30 age- and sex-matched controls in the first group. Results: In terms of AGA grades, grade 5 was the most common, followed by grades 4 and 2 each (20\% each), and finally grade 3. (16 percent ). Grades 1 and 6 were the least common, with only $10 \%$ of students achieving them. Those with metabolic syndrome (median $=6.38$, min- $\max =0.99-80)$ had substantially higher levels of alarin $(\mathrm{P}=0.001)$ than those without (median=1.08, min-max=0.22-8.37); A significant positive correlation was found between the Alarin level and triglycerides $(r=0.723)$, cholesterol $(r=0.603)$, LDL $(r=0.333 \&$ P-value $=0.015)$, VLDL $(r=0.466)$ and FBS $(r=$ $0.596 \&$ P-value $=0.001)$. Neither the alarin level nor HDL, age of illness start, length or systolic blood pressure showed any significant correlations AGA patients had substantially higher median Alarin levels than controls (0.95, IQR; 0.511.12) (5.58), IQR; (1.88-10.0), respectively). The P-value was below a threshold of 0.001 . P-value was 0.001 because the Alarin level revealed a significant variation among AGA grades; the meidan was 1.05 in grade 1, 1.88 in grade 2, 2.15 in grade 3, 6.38 in grade 4, and 11.0 in lesson 5 and 20.5 in grade 6 . Conclusion: Over $70 \%$ of adult males and $50 \%$ of adult females suffer from androgenetic alopecia (AGA), the most prevalent form of hair loss. This study's findings show that high levels of Alarin are linked to AGA, and that these levels rise as the severity of the illness increases. Additionally, Alarin levels were substantially greater in those with metabolic syndrome and were linked to a higher lipid profile in these patients. According to the results of the present research, another new discovery indicates that Alarin may be able to predict MS and AGA.
\end{abstract}

Key words: Alarin, Androgenetic Alopecia.

\section{Introduction}

Androgenetic alopecia is a common but treatable disease that affects men and women differently. It is caused by both hormonal and genetic influences. In the past, research have shown that AGA may have psychological side effects, such as sadness, poor selfesteem, a distorted self-image, and fewer and less pleasurable social interactions. Quality of life (QOL) deteriorates in AGA patients as the illness progresses. Counseling and therapy are thus critical in these situations [1].

Those who suffer from androgenetic alopecia have smaller hair follicles due to the loss of hair follicle stem cells or progenitor cells. The presence of androgens, as well as a hereditary susceptibility, have been identified as critical etiological variables in AGA [2].

Recent research shows a connection between AGA and cardiovascular disease (CVD), diabetes mellitus and hypertension dating back to the early 1990s (HT). Early development of AGA and severe coronary artery disease or metabolic syndrome have a significant correlation. We don't know everything about the link between AGA and obesity just yet [3].

In the last several decades, obesity and the metabolic syndrome have spread like wildfire across the globe, posing serious health and economic issues. According to some reports, Alarin is linked to increased caloric intake and weight gain. However, it's not known if high levels of Alarin in the blood correlate with insulin resistance or the metabolic syndrome [4].

The study's goal was to find out whether there was a link between high levels of serum alarin and androgenic alopecia, as well as obesity and the metabolic syndrome.

\section{Patients and Methods}

The case control study was conducted in the Outpatient Department of Dermatology Venereology and Andrology, at Benha University Hospital during the period from Jane 2021 to June 2021.

Type of study

Case control study

\section{Sample size}

A total of 80 male participants were included in this study, divided into 2 groups:

- First group; included 50 patients diagnosed with androgenic alopecia, Grade I-VII of the HamiltonNorwood classification.

- Second group; included 30 age and sex matched participants. 


\section{Inclusion criteria}

1.Patients with clinically diagnosed and dermoscopically confirmed AGA willing to participate in the study

2. Age is $\geq 18$ years.

\section{Exclusion Criteria}

1. Patients with co-existing alopecia other than AGA,

2. Patients undetermined diagnosis of hair loss,

3. Patients with any other dermatological disease,

4. Patients with presence of other factors or diseases that may cause hair loss,

5. Patients with prior use of finasteride, topical minoxidil,

6. Patients with Type 1 diabetes, liver cirrhosis, hepatic and renal failure, congestive heart failure or other disease

7.Patients undergoing chemotherapeutic agents or other drugs that may interfere with hair growth before their first presentation will be excluded.

\section{Ethical consideration}

The study protocol was approved by Ethical

Committee of Faculty of Medicine Department of Dermatology at Benha University. Written consent was taken for every participant.

\section{All patients were subjected to the following:}

\section{Full history taking}

- Including the demographic parameters including age, duration of alopecia, detailed family history, occupation, education, marital status, history of onset, smoking history, and sports life.

- The onset age of $\leq 35$ years was recommended as early-onset AGA.

\section{Complete clinical examination}

- Vital signs: heart rate, temperature respiratory rate and blood pressure

- Systolic BP $\geq 135 \mathrm{mmHg}$ and diastolic BP $\geq 85 \mathrm{mmHg}$ will be recommended as cutoff points for hypertension.

- Anthropometric measurements

- Including; weight, height, and waist circumference, and body mass index (BMI),

- Waist circumference (WC) levels of $>94 \mathrm{~cm}$ was recommended as cutoff point for abdominal obesity.

- Overweight was defined as BMI ranging from 23.0 to $26.0 \mathrm{~kg} / \mathrm{m}^{2}$ and obesity as BMI equal to or higher than $\geq 30 \mathrm{~kg} / \mathrm{m}^{2}$.

- BMI was calculated using the formula weight $(\mathrm{kg}) /$ height $\left(\mathrm{m}^{2}\right)$.

- Based on the diagnostic criteria of International Diabetes Federation (IDF-2005), waist circumference $>94 \mathrm{~cm}$ and at least two of the following criteria, triglyceride value $>150 \mathrm{mg} / \mathrm{dL}$ or specific treatment for this lipid abnormality, high density lipoprotein $<40 \mathrm{mg} / \mathrm{dL}$ or specific treatment for this lipid abnormality, blood pressure $\geq 130 / 85 \mathrm{mmHg}$ or antihypertensive treatment, and FBG $\geq 100 \mathrm{mg} / \mathrm{dL}$ or diagnosed diabetes mellitus, were accepted as metabolic syndrome.

\section{Dermatological assessment}

Severity of AGA was evaluated according to Norwood-Hamilton scale in men ${ }^{(5)}$. Men with preservation of the frontal hairline were recorded as presenting 'female pattern'.

Male-pattern baldness (Norwood-Hamilton scale)

- Type I: Minimal hair loss.

- Type II: Minor recession of the frontotemporal hairline

- Type IIIa: The area of recession of the frontotemporal region is almost vertical with the front portion of the ear

- Type IIIv: In this type, hair loss is primarily in the vertex with possibly some frontal recession

- Type IV: Severe hair loss, especially in the frontal and frontotemporal hair, and significant diffuse hair thinning over the vertex. There is a broad band that separates vertex and the top of hair

- Type V: Hair loss at the vertex region is still separated from the frontotemporal region but the division is much less distinct

- Type VI: The bridge of hair that once crossed the crown is now been lost with only sparse hair remaining

- Type VII: Only a narrow band of hair in a horseshoe shape survives on the sides and back of the scalp. [6]

\section{Laboratory investigations}

- Lipid parameters and FBG levels were studied after a 12-hour fasting period.

- Triglyceride level $>150 \mathrm{mg} / \mathrm{dL}$ and HDL level $<40 \mathrm{mg} / \mathrm{dL}$ will be recommended as cutoff for dyslipidemia.

- FBG level $\geq 100 \mathrm{mg} / \mathrm{dL}$ will be recommended as cutoff for impaired fasting glycemia.

- Complete blood count (CBC), serum iron, total iron binding capacity, serum ferritin.

\section{Measurements of adipokines}

Serum Alarin levels were measured with a commercial ELISA Kit following the manufacturer's protocol (Phoenix Pharmaceuticals, Inc., Belmont, CA, USA). The kit had a sensitivity of $0.08 \mathrm{ng} / \mathrm{ml}$ and a linear range of $0.08-0.78 \mathrm{ng} / \mathrm{ml}$. Intra-assay and interassay variations were $<10 \%$ and $<15 \%$, respectively. The ELISA kit had been validated by the dealer, showing high sensitivity and excellent specificity for detection of human Alarin, but no significant crossreactivity with other members of the galanin peptide family.

\section{Statistical analysis of the data}

Data were fed to the computer and analyzed using IBM SPSS software package version 20.0. (Armonk, NY: IBM Corp) Qualitative data were described using number and percent. The Kolmogorov-Smirnov test was used to verify the normality of distribution Quantitative data were described using range (minimum and maximum), mean, standard deviation, median and interquartile range (IQR). Significance of the obtained results was judged at $\mathrm{p}$-value $<0.001$. 


\section{Results}

There was no significant difference between both groups regarding age; P-value was 0.192.Table (1)

All the studied patients $(100.0 \%)$ showed a progressive course. The mean age of onset was $(26.5$ \pm 4.41 )years. The mean duration was 12.32 years, with a standard deviation of 9.11. Table (2)

The mean weight was significantly higher in cases $(88.26 \pm 12.71 \mathrm{~kg})$ compared to controls $(77.80 \pm 8.54$ $\mathrm{kg}$ ); P-value was $<00.1$. Also, mean BMI was significantly higher in cases $(29.67 \pm 3.80)$ than controls ( $25.8 \pm 1.61)$; P-value was $<0.001$. Furthermore, waist circumference was significantly higher in cases (102.78 $\pm 2.63)$ than controls $(88.73 \pm 1.84)$; P-value was $<0.001$. There was no significant difference between both groups regarding height $(\mathrm{P}$-value $=0.336)$. Table (3)

Grade 5 was the most frequent grade of AGA (24.0\%), followed by 4 and 2 (20.0\% for each), then grade $3(16 \%)$. The least frequent grades were 1 and 6 $(10.0 \%)$ for each. Table (4)

Triglycerides were significantly higher in cases (mean; $178.08 \pm 41.52$ ) compared to controls (99.07士 31.84); P-value was $<0.001$. Also, high triglycerides were significantly higher in cases $(40.0 \%)$ compared to controls $(0.0 \%)$; $\mathrm{P}$ value was $<0.001$.

Cholesterol was significantly higher in cases

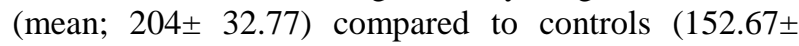
28.52); P-value was $<0.001$. Also, high cholesterol was significantly higher in cases $(16.0 \%)$ compared to controls $(0.0 \%)$; $\mathrm{P}$ value was $<0.001$.

As HDL was significantly lower in cases (mean; $33.64 \pm 4.15$ ) compared to controls (53.33 \pm 9.02); Pvalue was <0.001. Also, HDL above 60 was significantly lower in cases $(0.0 \%)$ compared to controls $(13.3 \%)$; $\mathrm{P}$ value was $<0.001$.

As LDL was significantly lower in cases (mean; $164 \pm 4.53)$ compared to controls $(129 \pm 11.98)$; P-value was <0.001. Also, high LDL (above 160) was significantly higher in cases $(92.0 \%)$ compared to controls $(3.3 \%)$; P value was $<0.001$.

VLDL was significantly lower in cases (mean; $31.7 \pm 3.55)$ compared to controls $(21.17 \pm 7.68)$; P-value was <0.001. Also, abnormal VLDL was significantly higher in cases $(62.0 \%)$ compared to controls $(3.3 \%)$; $\mathrm{P}$ value was $<0.001$. Table (5)

The median alarin level was significantly higher in cases (5.58), IQR; (1.88-10.0), compared to controls
(0.95), IQR; (0.51-1.12). P-value was $<0.001$. Table (6) \& figure (1)

Most of the patients $(82.0 \%)$ had metabolic syndrome. Table (7)

The mean FBS was significantly higher in cases $(95.32 \pm 14.32)$ compared to controls $(78.43 \pm 13.85)$; $\mathrm{P}=$ value was $<0.001$. Table $(8)$

Positive family history was significantly higher in cases $(72.0 \%)$ compared to controls $(33.3 \%)$; P-value was 0.001 . There was no significant difference between both groups regarding smoking $(\mathrm{P}$-value $=0.129)$. Table (9)

Systolic and diastolic blood pressure in both group .Table (10)

Alarin level was significantly higher in those with metabolic syndrome (median $=6.38, \min -\max =0.99-80$ ) compared to those without (median $=1.08$, min-max $=$ 0.22-8.37); P-value was <0.001. Table (11)

Alarin level showed significant positive correlations with triglycerised $(\mathrm{r}=0.723 \&$ \& -value < $0.001)$, cholesterol $(\mathrm{r}=0.603 \& \mathrm{P}$-value $<0.001)$, LDL $(\mathrm{r}=0.343 \&$ P-value $=0.015)$, VLDL $(r=0.466 \& \mathrm{P}-$ value $=0.001)$, duration $(\mathrm{r}=0.834 \& \mathrm{P}$-value $<0.001)$, weight $(\mathrm{r}=0.797 \&$ P-value $<0.001), \mathrm{BMI}(\mathrm{r}=0.847$ $\&$ P-value $<0.001)$, waist circumference $(r=0.518 \&$ Pvalue $<0.001)$, and FBS $(r=0.596 \&$ P-value $<0.001)$. There were no significant correaltions between alarin level and HDL, age of disease onset, height, or systolic blood pressure. Table (12)

ROC analysis was done for alarin level in diagnosing patients with alopecia. It showed a significant area under curve of 0.894 with a $95 \%$ confidence interval ranged between 0.819 to 0.969 (Pvalue $<0.001)$. The best cutoff was $>1.22$, at which sensitivity, specificity, PPV, NPV were $82.0 \%, 96.67 \%$, $97.6 \%$, and $76.3 \%$, respectively.Table (13 \& fig. 2)

ROC analysis was done for alarin level in predicting metabolic syndrome. It showed a significant area under curve of 0.854 with a $95 \%$ confidence interval ranged between 0.721 to 0.986 ( $\mathrm{P}$-value = $0.001)$. The best cutoff was $>2.16$, at which sensitivity, specificity, PPV, NPV were $75.61 \%, 88.89 \%, 96.9 \%$, and $44.4 \%$, respectively. Table (14\& fig. 3 )

There were statistically significant difference between Level of alarin and Grade of AGA in patients. Table (15)

Table (1) Comparison between the two studied groups according to age (years)

\begin{tabular}{lcccc}
\hline Age (years) & $\begin{array}{c}\text { Cases } \\
(\mathbf{n}=\mathbf{5 0})\end{array}$ & $\begin{array}{c}\text { Control } \\
(\mathbf{n = 3 0})\end{array}$ & $\mathbf{t}$ & $\mathbf{p}$ \\
\hline Min. - Max. & $24.0-62.0$ & $29.0-55.0$ & & \\
Mean + SD. & $40.84 \pm 9.43$ & $38.17 \pm 7.58$ & 1.317 & 0.192 \\
Median (IQR) & 40.50 & 35.0 & & \\
\hline
\end{tabular}

t: Student t-test

IQR: Inter quartile range $\mathrm{p}$ : $\mathrm{p}$ value for comparing between the studied groups SD: Standard deviation 
Table (2) Distribution of the studied cases according to different parameters in cases group $(\mathrm{n}=50)$

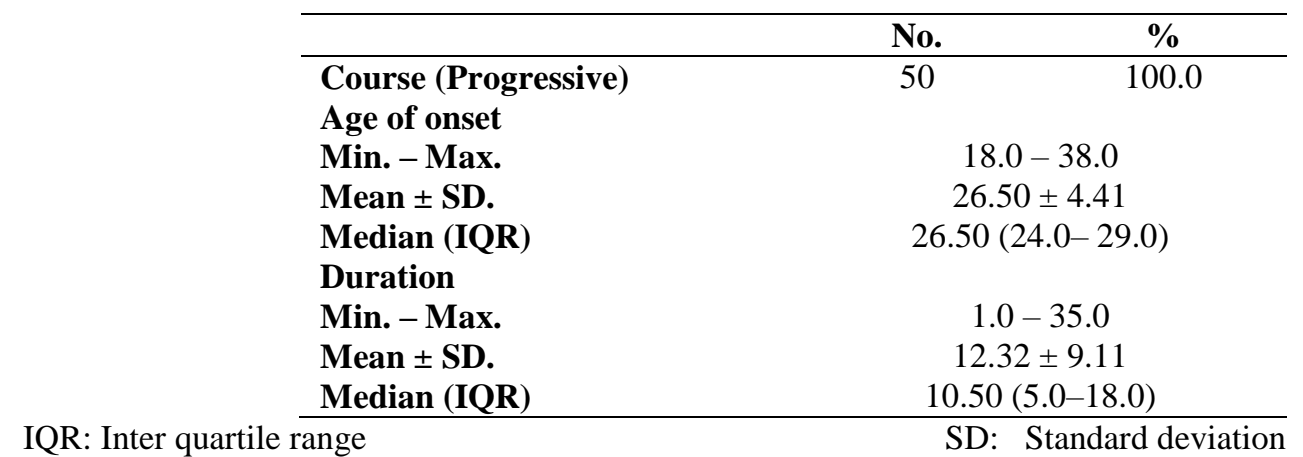

Table (3) Comparison between the two studied groups according to anthropometric measures

\begin{tabular}{|c|c|c|c|c|}
\hline & $\begin{array}{c}\text { Cases } \\
(n=50)\end{array}$ & $\begin{array}{l}\text { Control } \\
(n=30)\end{array}$ & $\mathbf{t}$ & $\mathbf{p}$ \\
\hline \multicolumn{5}{|l|}{ Weight } \\
\hline Min. - Max. & $65.0-113.0$ & $62.0-96.0$ & & \\
\hline Mean \pm SD. & $88.26 \pm 12.71$ & $77.80 \pm 8.54$ & $4.396^{*}$ & $<0.001^{*}$ \\
\hline Median (IQR) & $94.0(78.0-98.0)$ & $75.0(72.0-82.0)$ & & \\
\hline \multicolumn{5}{|l|}{ Length } \\
\hline Min. - Max. & $160.0-185.0$ & $160.0-188.0$ & & \\
\hline Mean \pm SD. & $172.16 \pm 5.95$ & $173.60 \pm 7.20$ & 0.968 & 0.336 \\
\hline Median (IQR) & $170.0(169.0-177.0)$ & $173.0(167.0-179.0)$ & & \\
\hline \multicolumn{5}{|l|}{ BMI } \\
\hline Min. - Max. & $22.50-36.10$ & $23.40-30.30$ & & \\
\hline Mean \pm SD. & $29.67 \pm 3.80$ & $25.80 \pm 1.61$ & $6.322^{*}$ & $<0.001^{*}$ \\
\hline Median (IQR) & $29.35(27.10-32.90)$ & $25.40(25.10-26.40)$ & & \\
\hline \multicolumn{5}{|c|}{ Waist Circumference } \\
\hline Min. - Max. & $94.0-108.0$ & $86.0-94.0$ & & \\
\hline Mean \pm SD. & $102.78 \pm 2.63$ & $88.73 \pm 1.84$ & $25.713^{*}$ & $<0.001^{*}$ \\
\hline Median (IQR) & $103.0(102.0-104.0)$ & $88.0(88.0-90.0)$ & & \\
\hline
\end{tabular}

\section{t: Student t-test}

*: Statistically significant at $\mathrm{p} \leq 0.05$

$\mathrm{p}: \mathrm{p}$ value for comparing between the studied groups

SD: Standard deviation

IQR: Inter quartile range

Table (4) Distribution of the studied cases according to the grade of aga in cases group $(n=50)$

\begin{tabular}{lcc}
\hline Grade of AGA & No. & \% \\
\hline $\mathbf{1}$ & 5 & 10.0 \\
$\mathbf{2}$ & 10 & 20.0 \\
$\mathbf{3}$ & 8 & 16.0 \\
$\mathbf{4}$ & 10 & 20.0 \\
$\mathbf{5}$ & 12 & 24.0 \\
$\mathbf{6}$ & 5 & 10.0 \\
\hline
\end{tabular}

Table (5) Comparison between the two studied groups according to lipid profile.

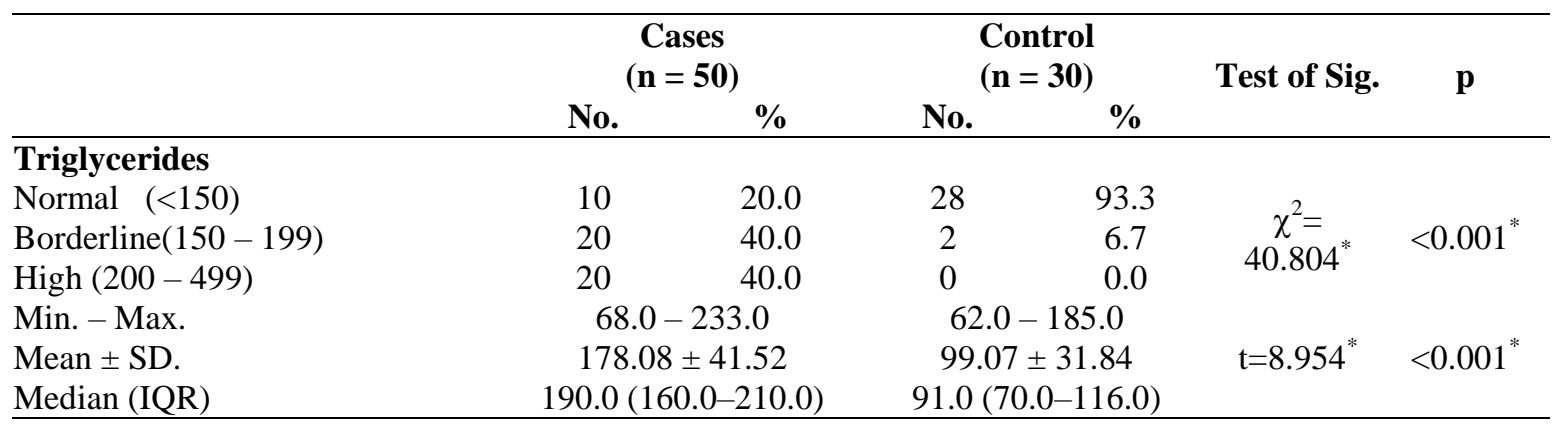




\begin{tabular}{|c|c|c|c|c|c|c|}
\hline \multicolumn{7}{|l|}{ Cholesterol } \\
\hline Desirable $(<200)$ & 15 & 30.0 & 28 & 93.3 & \multirow{3}{*}{$\begin{array}{c}\chi^{2}= \\
30.381^{*}\end{array}$} & \multirow{3}{*}{$<0.001^{*}$} \\
\hline Borderline (200 - 239) & 27 & 54.0 & 2 & 6.7 & & \\
\hline $\operatorname{High}(\geq 240)$ & 8 & 16.0 & 0 & 0.0 & & \\
\hline Min. - Max. & \multicolumn{2}{|c|}{$130.0-250.0$} & \multicolumn{2}{|c|}{$95.0-205.0$} & \multirow{3}{*}{$\mathrm{t}=7.115^{*}$} & \multirow{3}{*}{$<0.001^{*}$} \\
\hline Mean \pm SD & 204 & 2.77 & 152 & 8.52 & & \\
\hline Median (IQR) & 210.0 & $-225.0)$ & 150.0 & $-170.0)$ & & \\
\hline \multicolumn{7}{|l|}{ HDL } \\
\hline$<40$ & 40 & 80.0 & 2 & 6.7 & \multirow{3}{*}{$\begin{array}{c}\chi^{2}= \\
45.132^{*}\end{array}$} & \multirow{3}{*}{${ }^{{ }^{\mathrm{MC}} \mathrm{p}}$} \\
\hline Normal (40 - 60) & 10 & 20.0 & 24 & 80.0 & & \\
\hline$>60$ & 0 & 0.0 & 4 & 13.3 & & \\
\hline Min. - Max. & \multicolumn{2}{|c|}{$30.0-45.0$} & \multicolumn{2}{|c|}{$36.0-75.0$} & \multirow{3}{*}{$\mathrm{t}=11.266^{*}$} & \multirow{3}{*}{$<0.001^{*}$} \\
\hline Mean \pm SD & & .15 & & .02 & & \\
\hline Median (IQR) & 32.0 & $-36.0)$ & 52.0 & $60.0)$ & & \\
\hline \multicolumn{7}{|l|}{ LDL } \\
\hline Normal $(<130)$ & 0 & 0.0 & 17 & 56.7 & \multirow{3}{*}{$\begin{array}{c}\chi^{2}= \\
63.024^{*}\end{array}$} & \multirow{3}{*}{$<0.001^{*}$} \\
\hline Borderline $(130-159)$ & 4 & 8.0 & 12 & 40.0 & & \\
\hline High $(\geq 160)$ & 46 & 92.0 & 1 & 3.3 & & \\
\hline Min. - Max. & \multicolumn{2}{|c|}{$145.0-170.0$} & \multicolumn{2}{|c|}{$115.0-169.0$} & \multirow{3}{*}{$\mathrm{t}=14.330^{*}$} & \multirow{3}{*}{$<0.001^{*}$} \\
\hline Mean \pm SD & 16 & 4.53 & 131 & 1.98 & & \\
\hline Median (IQR) & 164.0 & $-168.0)$ & 129.0 & $-132.0)$ & & \\
\hline \multicolumn{7}{|l|}{ VLDL } \\
\hline Normal $(2-30)$ & 19 & 38.0 & 29 & 96.7 & \multirow{2}{*}{$\begin{array}{c}\chi^{2}= \\
26.889^{*}\end{array}$} & \multirow{2}{*}{$<0.001^{*}$} \\
\hline Abnormal & 31 & 62.0 & 1 & 3.3 & & \\
\hline Min. - Max. & \multicolumn{2}{|c|}{$22.0-39.0$} & \multicolumn{2}{|c|}{$5.0-31.0$} & \multirow{3}{*}{$\mathrm{t}=7.072^{*}$} & \multirow{3}{*}{$<0.001^{*}$} \\
\hline Mean \pm SD & & .55 & & .68 & & \\
\hline Median (IQR) & 32.0 & $-35.0)$ & 23.5 & $-28.0)$ & & \\
\hline
\end{tabular}

\section{$\chi^{2}$ : Chi square test MC: Monte Carlo}

$\mathrm{p}$ : $\mathrm{p}$ value for comparing between the studied groups

*: Statistically significant at $\mathrm{p} \leq 0.05$

\section{IQR: Inter quartile range SD: Standard deviation}

Table (6) Comparison between the two studied groups according to the level of alarin.

\begin{tabular}{lcccc}
\hline Level of alarin & $\begin{array}{c}\text { Cases } \\
(\mathbf{n = 5 0})\end{array}$ & $\begin{array}{c}\text { Control } \\
(\mathbf{n}=\mathbf{3 0})\end{array}$ & $\mathbf{U}$ & $\mathbf{p}$ \\
\hline Min. - Max. & $0.22-80.0$ & $0.19-9.39$ & & \\
Mean + SD. & $9.31 \pm 13.34$ & $1.09 \pm 1.60$ & $159.0^{*}$ & $<0.001^{*}$ \\
Median (IQR) & $5.58(1.88-10.0)$ & $0.95(0.51-1.12)$ & & \\
\hline hitney test & & p: $\mathrm{p}$ value for comparing between the studied groups \\
lly significant at $\mathrm{p} \leq 0.05$ & & IQR: Inter quartile range
\end{tabular}

U: Mann Whitney test
*: Statistically significant at $\mathrm{p} \leq 0.05$

SD: Standard deviation

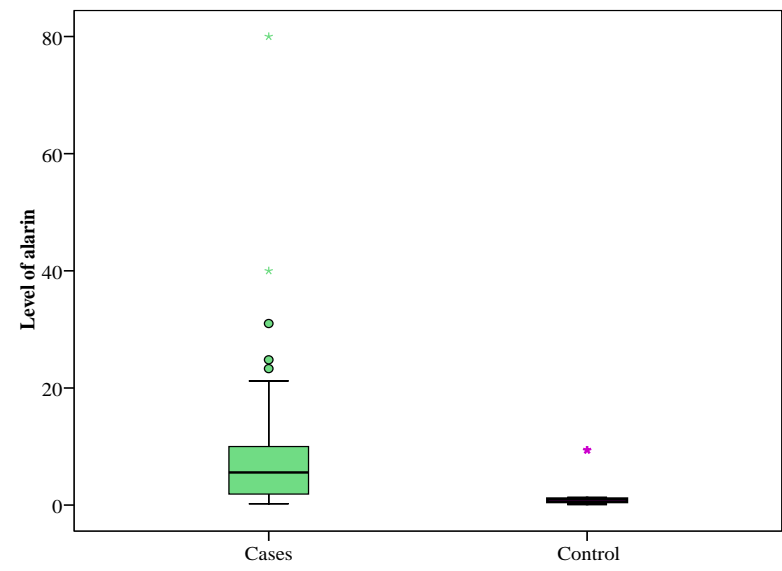

Fig. (1)Comparison between the two studied groups according to level of alarin. 
Table (7) Distribution of the studied cases according to different parameters in cases group $(\mathrm{n}=50)$

\begin{tabular}{rlcc}
\cline { 2 - 4 } & & No. & $\%$ \\
\cline { 2 - 4 } Metabolic Syndrome & & 9 & 18.0 \\
No & & 41 & 82.0 \\
\cline { 2 - 4 } Yes & SD: & Standard deviation
\end{tabular}

Table (8) Comparison between the two studied groups according to FBS

\begin{tabular}{lcccc}
\hline FBS & $\begin{array}{c}\text { Cases } \\
(\mathbf{n}=\mathbf{5 0})\end{array}$ & $\begin{array}{c}\text { Control } \\
(\mathbf{n = 3 0})\end{array}$ & $\mathbf{t}$ & $\mathbf{p}$ \\
\hline Min. - Max. & $50.0-120.0$ & $52.0-96.0$ & & \\
Mean + SD. & $95.32 \pm 14.32$ & $78.43 \pm 13.85$ & $5.169^{*}$ & $<0.001^{*}$ \\
Median (IQR) & $98.0(85.0-105.0)$ & $85.0(62.0-90.0)$ & & \\
\hline
\end{tabular}

t: Student t-test

p: $p$ value for comparing between the studied groups

*: Statistically significant at $\mathbf{p} \leq \mathbf{0 . 0 5}$

IQR: Inter quartile range

SD: Standard deviation

Table (9) Comparison between the two studied groups according to family history and smoking.

\begin{tabular}{|c|c|c|c|c|c|c|}
\hline & \multicolumn{2}{|c|}{$\begin{array}{c}\text { Cases } \\
(n=50)\end{array}$} & \multicolumn{2}{|c|}{$\begin{array}{l}\text { Control } \\
(n=30)\end{array}$} & \multirow[t]{2}{*}{$\chi^{2}$} & \multirow[t]{2}{*}{$\mathbf{p}$} \\
\hline & No. & $\%$ & No. & $\%$ & & \\
\hline \multicolumn{7}{|c|}{ Family History } \\
\hline Negative & 14 & 28.0 & 20 & 66.7 & \multirow{2}{*}{$11.471^{*}$} & \multirow{2}{*}{$0.001^{*}$} \\
\hline Positive & 36 & 72.0 & 10 & 33.3 & & \\
\hline \multicolumn{7}{|l|}{ Smoking } \\
\hline No & 27 & 56.3 & 22 & 73.3 & \multirow{2}{*}{2.307} & \multirow{2}{*}{0.129} \\
\hline Yes & 21 & 43.8 & 8 & 26.7 & & \\
\hline
\end{tabular}

$\chi 2$ : Chi square test

p: $p$ value for comparing between the studied groups

$*$ : Statistically significant at $\mathbf{p} \leq \mathbf{0 . 0 5}$

Table (10) Comparison between the two studied groups according to blood pressure.

\begin{tabular}{lcccc}
\hline & $\begin{array}{c}\text { Cases } \\
(\mathbf{n = 5 0 )}\end{array}$ & $\begin{array}{c}\text { Control } \\
(\mathbf{n = 3 0 )}\end{array}$ & $\mathbf{t}$ & $\mathbf{p}$ \\
\hline Systolic & $110.0-135.0$ & $90.0-125.0$ & & \\
Min. - Max. & $125.0 \pm 5.44$ & $112.50 \pm 9.54$ & $6.567^{*}$ & $<0.001^{*}$ \\
Mean \pm SD. & $125.0(120.0-130.0)$ & $115.0(110.0-120.0)$ & & \\
Median (IQR) & & & & \\
Diastolic & $60.0-85.0$ & $60.0-80.0$ & & \\
Min. - Max. & $80.50 \pm 6.94$ & $73.0 \pm 7.02$ & $4.657^{*}$ & $<0.001^{*}$ \\
Mean \pm SD. & $85.0(80.0-85.0)$ & $70.0(70.0-80.0)$ & & \\
Median (IQR) & & & &
\end{tabular}

\section{t: Student t-test}

$\mathrm{p}$ : $\mathrm{p}$ value for comparing between the studied groups

*: Statistically significant at $\mathrm{p} \leq 0.05$
IQR: Inter quartile range
SD: Standard deviation

Table (11) Relation Metabolic Syndrome and Level of alarin in patients $(n=50)$

\begin{tabular}{lcccc}
\hline \multirow{2}{*}{ Level of alarin } & \multicolumn{2}{c}{ Metabolic Syndrome } & \multirow{2}{*}{ Yes $(\mathbf{n = 4 1})$} & p \\
\hline Min. - Max. & $0.22-8.37$ & $0.99-80.0$ & \\
Mean \pm SD. & $1.96 \pm 2.51$ & $10.92 \pm 14.21$ & $54.0^{*}$ & $<0.001^{*}$ \\
Median & 1.08 & 6.38 & \\
\hline Whitney test & \multicolumn{5}{c}{ p: $p$ value for comparing between the studied groups } \\
cally significant at $\mathrm{p} \leq 0.05$ & \multicolumn{5}{c}{ SD: Standard deviation }
\end{tabular}

U: Mann Whitney test

*: Statistically significant at $\mathrm{p} \leq 0.05$ $\mathrm{p}$ : $\mathrm{p}$ value for comparing between the studied groups

Standard deviation 
Table (12) Correlation between serum level of alarin and different parameters in cases group $(n=50)$.

\begin{tabular}{lcc} 
& \multicolumn{2}{c}{ Level of alarin } \\
\cline { 2 - 3 } & \multicolumn{1}{c}{$\mathbf{r}_{\mathbf{s}}$} & $\mathbf{p}$ \\
\hline Cholesterol & 0.723 & $<0.001^{*}$ \\
HDL & 0.603 & $<0.001^{*}$ \\
LDL & -0.192 & $0.182^{*}$ \\
VLDL & 0.343 & $0.015^{*}$ \\
Age of onset & 0.466 & $0.001^{*}$ \\
Duration & -0.008 & 0.953 \\
Weight & 0.834 & $<0.001^{*}$ \\
Length & 0.797 & $<0.001^{*}$ \\
BMI & 0.179 & $0.2122^{*}$ \\
Waist Circumference & 0.847 & $<0.001^{*}$ \\
FBS & 0.518 & $<0.001^{*}$ \\
Systolic & 0.596 & $<0.001^{*}$ \\
Diastolic & 0.008 & 0.957 \\
$\mathrm{r}_{\mathrm{s}}$ : Spearman coefficient & 0.088 & 0.542 \\
\hline
\end{tabular}

Table (13) Validity (AUC, sensitivity, specificity) for Level of alarin to discriminate androgenetic Alopecia patients $(n=50)$ from control $(n=30)$

\begin{tabular}{|c|c|c|c|c|c|c|c|c|}
\hline & AUC & $\mathbf{p}$ & 95\% C.I & Cut off ${ }^{\#}$ & Sensitivity & Specificity & PPV & NPV \\
\hline Level of alarin & 0.894 & $<0.001^{*}$ & $0.819-0.969$ & $>1.22$ & 82.0 & 96.67 & 97.6 & 76.3 \\
\hline \multicolumn{3}{|c|}{$\begin{array}{l}\text { AUC: Area Under a Curve } \\
\text { NPV: Negative predictive value }\end{array}$} & \multicolumn{3}{|c|}{$\mathrm{p}$ value: Probability value } & \multicolumn{3}{|c|}{ CI: Confidence Intervals } \\
\hline \multicolumn{3}{|c|}{$\begin{array}{l}\text { NPV: Negative predictive value } \\
\text { \#Cut off was choose according to }\end{array}$} & \multicolumn{3}{|c|}{ PPV: Positive predictive value } & \multicolumn{3}{|c|}{$*$ : Statistically significant at $\mathrm{p} \leq 0.05$} \\
\hline
\end{tabular}

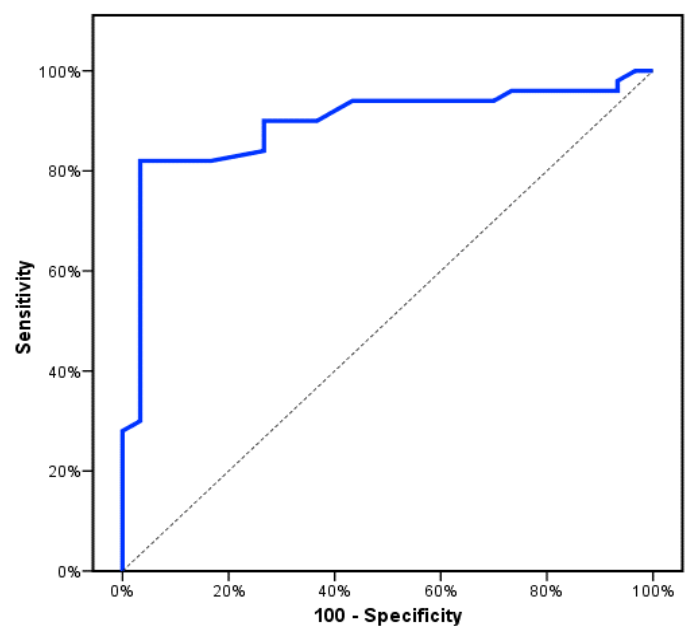

Fig. (2) ROC curve for Level of alarin to discriminate androgenetic Alopecia patients $(\mathrm{n}=50)$ from control $(\mathrm{n}=30)$

Table (14) Validity (AUC, sensitivity, specificity) for Level of alarin to predict Metabolic Syndrome patients $(\mathrm{n}=41)$ from non-metabolic Syndrome $(\mathrm{n}=9)$

\begin{tabular}{|c|c|c|c|c|c|c|c|c|}
\hline & AUC & p & 95\% C.I & Cut off ${ }^{\#}$ & Sensitivity & Specificity & PPV & NPV \\
\hline Level of alarin & 0.854 & $0.001^{*}$ & $0.721-0.986$ & $>2.16$ & 75.61 & 88.89 & 96.9 & 44.4 \\
\hline \multicolumn{3}{|c|}{ AUC: Area Under a Curve } & $\mathrm{p}$ value: $\operatorname{Pr}$ & ility value & \multicolumn{4}{|c|}{ CI: Confidence Intervals } \\
\hline \multicolumn{3}{|c|}{ NPV: Negative predictive value } & PPV: Positi & redictive $\mathrm{v}$ & \multicolumn{4}{|c|}{$*$ : Statistically significant at $p \leq 0.05$} \\
\hline
\end{tabular}




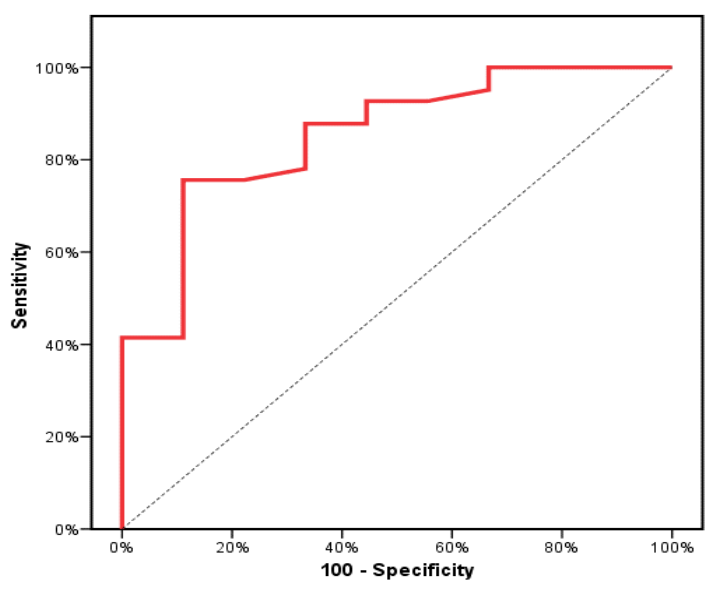

Fig. (3) ROC curve for Level of alarin to predict Metabolic Syndrome patients $(n=41)$ from non-metabolic Syndrome $(n=9)$

Table (15) Relation between Level of alarin and Grade of AGA in patients $(n=50)$

\begin{tabular}{llccccc}
\hline Grade of AGA & $\mathbf{N}$ & Min. - Max. & $\begin{array}{c}\text { Level of alarin } \\
\text { Mean } \pm \text { SD. }\end{array}$ & Median & H & p \\
\hline 1 & $\mathbf{5}$ & $0.25-1.08$ & $0.81 \pm 0.36$ & 1.05 & & \\
2 & $\mathbf{1 0}$ & $0.22-3.66$ & $1.74 \pm 0.96$ & 1.88 & & \\
3 & $\mathbf{8}$ & $1.25-4.09$ & $2.36 \pm 0.85$ & 2.15 & & $<0.001^{*}$ \\
4 & $\mathbf{1 0}$ & $5.0-10.0$ & $6.98 \pm 1.64$ & 6.38 & $43.036^{*}$ & \\
5 & $\mathbf{1 2}$ & $8.18-80.0$ & $20.72 \pm 20.88$ & 11.0 & & \\
6 & $\mathbf{5}$ & $10.40-31.0$ & $21.34 \pm 7.54$ & 20.50 & & \\
\hline
\end{tabular}

$\mathrm{H}: \mathrm{H}$ for Kruskal Wallis test

*: Statistically significant at $\mathrm{p} \leq 0.05$

$\mathrm{p}$ : $\mathrm{p}$ value for comparing between Level of alarin and Grade of AGA

\section{Discussion}

The research was carried out at the outpatient clinic of the Benha University Hospitals' Dermatology and Andrology and Venereology Department during the study year 2020. There were a total of 80 men in the study, divided into the following two categories: Group one consisted of 50 men with androgenic alopecia (Grades I-VII of the Hamilton-Norwood classification [7] and 30 healthy men as controls.

According to the data, the average age of the participants was 40.84 years, with no significant difference in age between the two groups (P-value: 0.192). One hundred and zero percent of the individuals examined had a steadily worsening condition. In this study, the average age of onset was 26.5 years, and the standard deviation was 4.41 years. The average time was 12.32 years, although there was a 9.11-year spread.

According to Shankar et al. [8], a population-based research of 1,005 males found that our findings matched theirs. One hundred and five males were studied in all, with a mean age of 37.05 and a standard deviation of 4.79. The youngest participant was 30 years old, and the oldest was 49 years old. $2 \%$ of the participants were between the ages of 30 and 35, 34.4 percent were between the ages of 36 and 40, 26.0 percent were between the ages of 41 and 45 , and 0.4 percent were between the ages of 46 and 50 .
Ozbas Gok et al., [9], who examined the link between androgenetic alopecia and metabolic syndrome, looked at 74 male AGA patients (aged 20-50 years, mean 32.14) and 42 controls (aged 20-50 years, mean 34.40).

According to Su and Chen's research [10], which examined the link between androgenetic alopecia and smoking, as well as the prevalence of smoking among Asian males. This poll drew the participation of 740 of the 924 males that were invited. Participants' average age was 65.2 years, with a standard deviation of 11.2 years (age range, 40-91 years).

The mean weight of patients was substantially greater than that of controls in this research, with a Pvalue of 00.1. Additionally, mean BMI was substantially greater in cases compared to controls, with a P-value of 0.001 . The waist circumference of those who had the condition was considerably larger than that of the controls $(\mathrm{P}=0.001)$. In terms of length, there was no significant difference between the two groups $(\mathrm{P}=0.336)$.

Studies on androgenetic alopecia, the metabolic syndrome, and insulin resistance have shown similar findings. Is there a link between the two? This was a case-control study. P 0.001, $\mathrm{P}=0.002$, waist circumference, and BMI were all statistically different between the patients and controls (P 0.001), and these changes were statistically significant. 
Androgenetic alopecia was shown to be associated with metabolic syndrome by Kumar et al. [12]. There were no significant differences between the AGA group and the controls in terms of participants' weight, height, or BMI in the study by Ozbas Gok et al. (9), but there were statistically significant differences in waist circumference between the cases $(45.31 \%)$ and the controls $(12.5 \%), \mathrm{P}=0.022$. The waist circumference in the control group was also substantially higher $(\mathrm{p}=0.044)$. In previous research, individuals with severe alopecia were included, but in this study, only those with moderate alopecia were.

This research looked at AGA grades, and found that the fifth grade had the highest percentage of AGA students $(24 \%)$, followed by grades four and two each with $20 \%$ of students, and finally grade three with $14 \%$ of students (16 percent ). Grades 1 and 6 were the least common, with only $10 \%$ of students achieving them.

One in four (24 percent) AGA patients were in stage 2,26 percent $(35.1 \%)$ were in stage 3 , and 23 percent ( 23 percent) were in stage $3 \mathrm{~V}$, according to the Hamilton-Norwood classification. Only one patient (1.4 percent) was in stage 5, and six $(8.1 \%)$ were in stage 7 .

This research found that 27.27 percent of participants had grade II alopecia, whereas 22.12 percent had grade I alopecia, and the remaining 3.17 percent had grade III alopecia (21.78 percent)

A substantial difference existed between the patients and controls in this research, with Triglycerides being considerably higher in the cases. The P-value was 0.001 . The percentage of patients with elevated triglycerides $(40.0 \%)$ was considerably greater than the percentage of controls $(0.0 \%)$; $\mathrm{P}$ value 0.001 . P-value 0.001 indicated that cholesterol levels were substantially higher in patients compared to controls. Also, the prevalence of elevated cholesterol was considerably greater in cases $(16.0 \%)$ than in controls $(0.0 \%)$, with a $\mathrm{p}$ value of 0.001 (significant difference). P-value 0.001 indicated that HDL levels were substantially lower in patients than in controls. There was a significant difference between the cases and controls in the percentage of those with HDL cholesterol levels more than $60 \quad(\mathrm{P}=0.001)$. When comparing patients to controls, LDL was substantially lower in the former group; P-value was 0.001 . There was also a significant increase in the percentage of patients with high LDL (over 160) as compared to controls (3.3 percent); $\mathrm{P}$ value was 0.001 . When comparing cases to controls, VLDL levels were substantially lower in the former $(\mathrm{P}=0.001)$. VLDL levels were also abnormally high (62.0 percent) compared to controls (3.3 percent); $\mathrm{P}$ value 0.001 .

Our findings matched those of Bakry et al. [11], who found that TGs were considerably higher (p0.001) and HDL-C was significantly lower (p0.001) in study patients than in control individuals. Kumar et al. [12] observed that the AGA group had significantly higher serum TG levels than the normal controls.

An increase in AGA patients' blood androgen levels is associated with the proliferation of smooth muscle cells inside arteries and a reduction in HDL-C levels, both of which have been seen experimentally. Atheroma to atherothrombosis conversion was linked to low HDL$\mathrm{C}$ and high TG levels. To minimise this danger, it's critical to look at lipid profiles in AGA patients. [12]

A total of 128 people with early AGA were included in the research conducted by Acibucu et al. [13]. Eighty male participants were in the sick group, while fortyeight healthy male subjects were in the control group. The changes in TG and total cholesterol between the groups were statistically significant (p 0.05).

However, Ozbas Gok et al. (9), found no significant changes in participants' TG or HDL between the AGA group and controls ( $\mathrm{p}=0.306$ and $\mathrm{p}=0.779$, respectively). Other confounding variables, such as family history and smoking status, may have contributed to this discrepancy, although they were not fully controlled. This may be because their research excluded older individuals and the majority of patients had moderate alopecia in comparison to the other studies. This could be the reason

The metabolic syndrome was seen in the majority of individuals in this research (82.0 percent) (MS).

When compared to controls (17\%), the prevalence of MS was statistically very highly significant (P 0.001), and Bakry et al. [11], MS was diagnosed in 39 cases and 19 controls, with a significant difference between the two groups $(\mathrm{P}=0.002)$. Our findings matched those of Kumar et al. [12].

AGA had a $14.9 \%$ MS prevalence compared to a control group's $26.2 \%$, according to Ozbas Gok et al. [9], although the difference was not statistically significant $(\mathrm{p}=0.135)$. There may be a correlation between the low incidence of MS and the study's exclusion of older individuals, as well as the fact that the majority of patients had moderate alopecia in comparison to previous studies.

An individual with the metabolic syndrome (MS) has a higher risk of cardiovascular disease. A number of variables are implicated in the aetiology of MS, including genetic susceptibility, insulin resistance (IR), obesity, hypertension, dyslipidemic disorders, vascular abnormalities, inflammation, hyperandrogenism, low uric acid levels, and a lack of vitamin D. [14].

Second, hormonal changes like hyperandrogenism may play a role in the development of AGA and hypertension. These hypotheses have been put forth to explain the connection between AGA and MS. AGA and cardiovascular disease are both linked to excess androgens, as have both AGA and cardiovascular disease [15].

A substantial number of early AGA patients (80) had IR or MS, according to a research by Acibucu et al. In 50 male patients with grade 3 AGA, Mumcuoglu et al. [16] discovered a link between AGA and IR, but not MS. It's unclear how insulin works on the AGA. Researchers believe insulin, which causes vasoconstriction and nutritional deprivation, may have a role in the development of AGA. It's also possible that it works by making testosterone work better. In obese 
individuals, insulin and insulin-like growth factor-1 may increase DHT levels by activating 5--reductase [17].

Compared to the controls, cases had substantially higher mean FBS; $\mathrm{P}=$ value was 0.001. Our findings matched those of Bakry et al. [11], who found statistically significant differences between patients and controls in terms of mean FBS levels (P 0.001). Control group had substantially higher FBG than AGA group than Ozbas Gok et al. [9], p0.001. It was decided that these surprising outcomes were just coincidental.

A greater percentage of patients (72.0 percent) had a positive family history compared to controls (33.3 percent); the P-value was 0.001 . Also, in a hospitalbased cross-sectional research in Turkey conducted by Salman et al. (18), $78.28 \%$ of the patients $(n=328)$ had a history of androgenetic alopecia (AGA), which was defined as hair loss caused by androgens. Men were more likely to have AGA if their father, brother, or other close family member had the disease, but there was no link found between male AGA prevalence and female AGA history.

According to $\mathrm{Su}$ and Chen [10], early-onset AGA was associated with a family history of the disease (odds ratio [OR]: 2.89; 95\% confidence interval [CI]: 1.42-5.88]. Moderate or severe AGA risk rose with the degree of family history (OR, 13.38; 95\% CI, 4.8037.27), second degree [OR, 6.33; 95\% CI, 2.37-16.91], and third degree [OR, 5.32; 95\% CI, 0.80-35.48]. There was a statistically significant link between moderate or severe AGA and a family history of AGA on the father's side (OR, 12.69; 95\% CI, 4.65-34.60), but not in the mother's side (OR, 3.07; 95 percent CI, 0.30-31.32).

Wang et al. [19] examined the prevalence of androgenetic alopecia in six Chinese cities: a community-based research, and found that $29 \%$ of men and $19 \%$ of women with AGA had a positive family history. It's unclear why the two people are so different, but genetic diversity is a possibility.

When it came to smoking, there was no significant difference between the two groups $(\mathrm{P}=0.129)$. While $\mathrm{Su}$ and Chen [10], who found that smokers had a higher risk of moderate or severe AGA (Norwood categories IV) in the univariate analysis (OR, 1.61; 95 percent CI, 1.05-2.46). In spite of this, no statistically significant links were found between the intensity or duration of smoking and AGA (OR, 1.77; 95\% CI, 1.14-2.76), current smoking of 20 or more cigarettes per day (OR, 2.34; 95\% CI, 1.19-4.59), smoking intensity (OR, 1.8; 95\% CI, 1.03-3.07), and dyslipidemia (OR, 1.47; 95\% CI, 1.01-2.14) after adjusting for $\mathrm{f}$.

Cases had substantially higher mean systolic and diastolic blood pressures than controls in this research. Each had a P-value of 0.001 . There were statistically significant differences between patients and controls in terms of mean systolic (P 0.001) and diastolic (P 0.001) blood pressure readings.

In Ozbas Gok et alresearch .'s [9], there were no significant differences in diastolic pressure readings between the AGA group and controls $(p=0.415)$. Nevertheless, both the AGA and control groups $(\mathrm{p}=0.043)$ had substantially higher systolic blood pressure than the former. Also, the difference in systolic blood pressure between patients and controls was statistically significant in the research by Kumar et al. [12]. However, there was no discernible difference between the groups in terms of diastolic blood pressure.

The artery wall endothelium contains androgenmediated receptors. With an increase in serum androgen levels in AGA patients, smooth muscle cells proliferate in arteries, raising blood pressure risk [20].

There was a significant difference in Alarin levels between individuals who had metabolic syndrome and those who did not; the study's P-value was 0.001 .

Galanin neuropeptides have been implicated in the control of hunger, insulin resistance, obesity, hypertension, and metabolism, according to previous research [21].

An alarin neuropeptide family member recently identified in the ganglion cells of neuroblastoma, alarin was originally discovered in the galanin family. The locus coeruleus, arcuate nucleus, olfactory bulb, and amygdala have all been shown to contain Alarin in follow-up investigations. The injection of alarin stimulation to the cerebral ventricle and peripheral areas of rats has been found to lower blood glucose levels in T2DM animals, increase skeletal muscle glucose uptake, and improve insulin resistance in the animals. [22]

All the evidence points to the role of insulin in controlling blood sugar levels, which in turn affects the onset and progression of diabetes mellitus. For instance, the hormone leptin, which reduces hunger, may also reduce insulin production. To regulate glucose and lipid metabolism and play a role in diabetes and obesity, insulin secretion may help stimulate leptin secretion [23].

The T2DM obese group had significantly higher plasma alarin levels than the T2DM non-obese group (obese T2DM; 1.030.34, VS non obese T2DM; 0.550.22, VS Control; 0.210.12, P0.05), according to a study done by Zhou et al. [24], who examined plasma Alarin levels and their influencing factors in obese newly diagnosed type 2 diabetes patients.

They also found that in MS patients, the levels of circulating Alarin were substantially higher than in healthy individuals in the research by Fang et al. [25], which investigated whether high circulation alarin levels are linked to the presence of metabolic syndrome

Researchers found strong links between Alarin level and triglycerides, cholesterol (LDL and VLDL), LDL (bad) and VLDL (good) in this research. Neither the alarin level nor HDL, age of illness start, length or systolic blood pressure showed any significant correlations

In agreement with Zhou et al. [24], correlation analysis showed that plasma alarin levels in T2DM patients were positively correlated with waistline and BMI as well as with TC and LDL-C as well as with FPG, HbA1c, FINS and HOMA-IR (P 0.05). They were also negatively correlated with HDL-C and HOMA- (P 
0.05), with T2DM obese individuals having a significantly higher correlation coefficient. The correlation coefficient In T2DM non-obese and T2DM obese patients, multiple linear stepwise regression analysis showed that BMI, FPG, HbA1c, HOMA-, and HOMAIR were independent factors related to plasma alarin levels and the correlation coefficient of the T2DM obese group was significantly higher than the T2DM non-obese group (P 0.05).

AUCglucose and TNF were positively associated with circulating Alarin levels in a research done by Fang et al. [25], which also found a correlation between circulating Alarin levels and WC, blood pressure, FBG, lipid, and haemoglobin A1c. The results of multivariate logistic regression showed a connection between MS and insulin resistance and levels of circulating Alarin.

The Alarin level was put through a ROC analysis to see whether it could be used to predict metabolic syndrome. P-value $=0.001$ ), the area under the curve was significant at 0.854 , and the 95 percent confidence interval spanned from 0.721 to 0.986 . The best cutoff was >2.16, which had a sensitivity, specificity, PPV, and NPV of 75.61 percent, 88.89 percent, and 96.9 percent, respectively, of 88.89 percent.

C-reactive protein (CRP) levels in the blood were shown to be predictive of both multiple sclerosis (MS) and insulin resistance in the research population conducted by Fang et al. However, because of sample size and an abnormal distribution of Alarin levels in the population, the AUC range (0.7-0.711) was deemed of mild-to-moderate significance.

For AGA (5.58), IQR; 1.88-10.0), the median Alarin level was considerably higher than for controls (0.95), IQR; 0.51-1.12) in the current research The Pvalue was below a threshold of 0.001 . P-value was 0.001 because the Alarin level revealed a significant variation among AGA grades; the meidan was 1.05 in grade 1, 1.88 in grade 2, 2.15 in grade 3, 6.38 in grade 4 , and 11.0 in lesson 5 and 20.5 in grade 6 . The level of alarin was subjected to ROC analysis.

\section{Conclusion}

Over $70 \%$ of adult males and $50 \%$ of adult females suffer from androgenetic alopecia (AGA), the most prevalent form of hair loss. This study's findings show that high levels of Alarin are linked to AGA, and that these levels rise as the severity of the illness increases. Additionally, Alarin levels were substantially greater in those with metabolic syndrome and were linked to a higher lipid profile in these patients. A recent research also discovered a new finding: Alarin may be able to predict MS and AGA.

\section{References}

[1] S.Gupta, I.Goyal, A.Mahendra, Quality of Life Assessment in Patients with Androgenetic Alopecia. Int J Trichology.vol.11, pp.147-152, 2019.

[2] S.Heilmann, D.Nyholt, F.Brockschmidt, et al. No genetic support for a contribution of prostaglandins to the aetiology of androgenetic alopecia. $\mathrm{Br} \mathrm{J}$ Dermatol.vol.169,pp.222-224, 2013.

[3] L.Su, L.Chen, S.Lin, et al. Association of androgenetic alopecia with mortality from diabetes mellitus and heart disease. JAMA Dermatol.vol.149, pp.601-606, 2013.

[4] M.Ng, T.Fleming, M.Robinson, et al. Global, regional, and national prevalence of overweight and obesity in children and adults during 19802013: a systematic analysis for the Global Burden of Disease Study 2013. Lancet.vol.384,pp.66-81,2014.

[5] OT.Norwood, Incidence of female androgenetic alopecia (female pattern alopecia) Dermatol Surg, vol. 27, no. 1, pp. 53-54, 2001.

[6] C.T.Wirya, W.Wu, and K.Wu, "Classification of male-pattern hair loss", International Journal of Trichology, Wolters Kluwer--Medknow Publications.vol. 9, pp.95,2017.

[7] E.Ludwig, Classification of the types of androgenetic alopecia (common baldness) occuring in the female sex. $\mathrm{Br} J$ Dermatol.vol.97,pp.247-254, 1977.

[8] D.S.K.Shankar, M.Chakravarthi, and R.Shilpakar, "Male androgenetic alopecia: population-based study in 1,005 subjects", International Journal of Trichology, Wolters Kluwer--Medknow Publications.vol.1, pp.131,2009.

[9] S.Ozbas Gok, A.Akin Belli, and E.Dervis, "Is there really relationship between androgenetic alopecia and metabolic syndrome?", Dermatology Research and Practice, Hindawi,2015.

[10] L.-H.Su band T.H.-H.Chen, "Association of androgenetic alopecia with smoking and its prevalence among Asian men: a communitybased survey", Archives of Dermatology, American Medical Association.vol.143, pp.1401-1406,2007.

[11] O.A.Bakry, M.A.M.Shoeib, M.K.El Shafiee and A.Hassan, "Androgenetic alopecia, metabolic syndrome, and insulin resistance: Is there any association? A case-control study", Indian Dermatology Online Journal, Wolters Kluwer--Medknow Publications.vol. 5, pp.276,2014.

[12] K.C.D.Kumar, Y.H.K.Kumar and V.Neladimmanahally, "Association of androgenetic alopecia with metabolic syndrome: A case-control study on 100 patients in a tertiary care hospital in South India", Indian Journal of Endocrinology and Metabolism, Wolters Kluwer--Medknow Publications.vol.22, pp.196,2018.

[13] F.Acibucu, M.Kayatas, F.Candan, "The association of insulin resistance and metabolic syndrome in early androgenetic alopecia", Singapore Medical Journal.vol.51, pp.931,2010. 
[14] B.Falkner, N.D.F.H.Cossrow, "Prevalence of metabolic syndrome and obesity-associated hypertension in the racial ethnic minorities of the United States", Current Hypertension Reports, Springer.vol.16, pp.449,2014.

[15] N.Trieu and G.D. Eslick, "Alopecia and its association with coronary heart disease and cardiovascular risk factors: a meta-analysis", International Journal of Cardiology, Elsevier.vol. 176, pp.687-695,2014.

[16] C.Mumcuoglu, T.R.Ekmekci and U.Sema, "The investigation of insulin resistance and metabolic syndrome in male patients with earlyonset androgenetic alopecia", European Journal of Dermatology.vol. 21, pp.79-82,2011.

[17] C.-C.Yang, F.-N.Hsieh, L.-Y.Lin, C.-K.Hsu, H.-M.Sheu and W. Chen, "Higher body mass index is associated with greater severity of alopecia in men with male-pattern androgenetic alopecia in Taiwan: a cross-sectional study", Journal of the American Academy of Dermatology, Elsevier.vol.70, pp.297-302,2014.

[18] K.E.Salman, I.K.Altunay, N.A.Kucukunal and A.A.Cerman, "Frequency, severity and related factors of androgenetic alopecia in dermatology outpatient clinic: hospital-based cross-sectional study in Turkey", Anais Brasileiros de Dermatologia, SciELO Brasil.vol.92, pp.3540,2017.

[19] T.L.Wang, C.Zhou, Y.W.Shen, X.Y.Wang, X.L.Ding, S.Tian, Y.Liu, et al. "Prevalence of androgenetic alopecia in China: a communitybased study in six cities", British Journal of Dermatology, Wiley Online Library.vol.162, pp.843-847,2010.

[20] J.-J.Cai, J.Wen, W.-H.Jiang, J.Lin, Y.Hong and Y.-S.Zhu, "Androgen actions on endothelium functions and cardiovascular diseases", Journal of Geriatric Cardiology: JGC, Institute of Geriatric Cardiology, Chinese PLA General Hospital.vol.13, pp.183,2016.

[21] Q.Yang, A.Vijayakumar and B.B.Kahn, "Metabolites as regulators of insulin sensitivity and metabolism", Nature Reviews Molecular Cell Biology, Nature Publishing Group.vol.19, pp.654-672,2018.

[22] A.Mikó, N.Füredi, J.Tenk, I.Rostás, S.Soós, M.Solymár, M.Székely, et al. "Acute central effects of alarin on the regulation on energy homeostasis", Neuropeptides, Elsevier.vol.64, pp.117-122,2017.

[23] N.Katsiki, D.P.Mikhailidis and M.Banach, "Leptin, cardiovascular diseases and type 2 diabetes mellitus", Acta Pharmacologica Sinica, Nature Publishing Group.vol.39, pp.1176$1188,2018$.

[24] X.Zhou, M.Luo, S.Zhou, Z.Cheng, Z.Chen and $\mathrm{X} . \mathrm{Yu}$, "Plasma Alarin Level and Its Influencing Factors in Obese Newly Diagnosed Type 2 Diabetes Patients", Diabetes, Metabolic
Syndrome and Obesity: Targets and Therapy, Dove Press.vol.14, pp.379,2021.

[25] P.Fang, L.Zhang, M.Yu, et al. Activiated galanin receptor 2 attenuates insulin resistance in skeletal muscle of obese mice. Peptides.vol.99, pp.92-98,2018.

[26] L.Guo, P.Fang, M.Yu, M.Shi, P.Bo and Z.Zhang, "Central alarin ameliorated insulin resistance of adipocytes in type 2 diabetic rats.", The Journal of Endocrinology.vol.223,pp.217225,2014. 